reducing the system; thereby relaxing the muscles, and with the hope of diminishing the severity of the consecutive inflammation. In this case, the subsequent inflammation was much less severe, and more easily controlled than that of William Joyner, which is attributed to the previous and more graduated reduction of the tone of the system, and justifies the course adopted and its desirableness in such cases.

It is hoped the success which has attended this attempt to reduce luxations of long-standing, will induce the profession to make similar efforts and not abandon patients (generally of the lower orders) to a life of decrepitude and dependence.

\section{POISONING FROM LUCIFER MATCHES.}

\section{TO THB BDITORS OF THE PROVINCIAL MEDICAL} JOURNAL.

Gentremen,-Cases of poisoning by lucifer matches being exceedingly rare, perhaps the following may prove interesting to the readers of your Journal.

On November 30, Mr. Young, house-surgeon to the Leicester Dispensary, requested me to assist him in examining the body of a little girl, aged six months, who five days previously had sucked the end of several lucifer matches, commonly called "Albert and Victoria." The following are the symptoms as given me by Mr. Denton, the medical attendant, and the parents.

About eight hours after sucking the matches, violent sickness came on, which continued during a night and day; the matter ejected being luminous and, as well as the breath, emitting a strong phosphorescent odor. After the cessation of the sickness, the child lay in a drowsy, stupid, and comatose condition, till within a few hours of its death, when it was seized with convulsions, and died in one of the paroxysms. The bowels, during the whole time, were sluggish, requiring frequent exhibition of enemata to cause evacuations.

Post-Mortem Appearances Twelve Hours after Death.

On opening the abdomen, the peritoneum presented nothing abnormal. Near the pyloric orifice of the stomach a large injected patch was seen, the mucous membrane being thickened and softened. The duodedenum and jejunum were quite in a healthy state; along the whole course of the ilium several oblong patches, from half an inch to three inches in length, attracted notice, the mucous membrane being thickened, softened, and in many places ulcerated; the colon and rectum were healthy; the liver was larger, paler, and more indurated than natural; the lungs and pleura were healthy; the pericardium contained from half to one ounce of fluid; neither the brain nor œsophagus were examined.

Your obedient servant,

R. A. Lafargue, M.R.C.S.L.

Leicester, Dec. 16, 1842.

\section{ON STAPHYLORAPHY IN CASES OF CLEFT PALATE.}

By M. Roux, Paris.

The velum palati, like the upper lip, may be affected by a species of congenital malformation, which consists in fissure or separation of the lateral portions, along the median line. This division may be simple -that is, confined to the soft parts ; or complicated that is, extending more or less to the hard palate, or even comprising the alveolar processes of the superior maxillary bone and the upper lip. Artificial reunion of the velum palati has been chiefly applied to the congenital disease; but it is equally suited to accidental malformations arising from ulceration, wounds of the mouth, or certain operations for the extraction of polypi, foreign bodies in the pharynx, \&c.

In some cases the disease is confined to simple bifurcation of the uvula; in others, which is less common, a part of the soft palate only is divided; in ordinary cases the fissure extends to the whole of the soft palate. M. Roux lately saw rather a rare example in the son of a German physician; the soft and hard palates were separated, but there was no hare-lip ; the velum palati, however, was imperfectly united by a thin transparent membrane, evidently formed by the mucous membrane. Although the fissure always exists on the median line the two sides of the palate do not always present the same degree of thickness or breadth-a circumstance which may cause some trouble in fixing the needles properly. On the other hand the palate may be in various states which are more or less favorable for the success of the operation. It may be solid, thick, and capable of supporting the sutures well; or it may be thin, membranous, and semitransparent. The degree of separation varies much; sometimes it is produced by simple bifurcation of the posterior nasal spine; sometimes by complete separation of the two sides of the roof of the mouth. In the latter case the cavity of the mouth communicates freely with that of the nares and pharynx.

As to solutions of continuity arising from wounds, we can readily conceive that they must be extremely irregular and of various forms, according to the causes which have produced them. The same remark applies to deformities from syphilis or scrofulous ulcerations, and the latter are much more frequent than has hitherto been supposed, their ravages being errone. ously attributed to secondary syphilis.

Having thus briefly noticed the anatomical varieties of cleft palate, it may be well to enumerate, as briefly as possible, the inconveniencies which arise from this deformity. In new-born children it gives rise to great difficulty, or even impossibility, of drawing the breast, according to the extent of the fissure. When the velum palati alone is cleft the child may be able to suct; but even then, whenever he is placed in a horizontal position, deglutition becomes extremely difficult; some assistance, however, may be rendered by placing the infant erect, and by pressing the bosom. When the hard palate and lip are divided the child is incapable of sucking, and must be supported by artificial means. As the individual advances in life much inconvenience may arise from a difficulty of pronouncing words intelligibly, and the voice is always nasal and disagreeable; certain guttural sounds can never be produced. The English physician on whom M. Roux performed his first operation pronounced the th like $s$, and spoke more distinctly in French than in English. "Verba naribus egredientia videbantur." Amongst minor inconveniencies may be reckoned the impossibility of blowing, of drinking in the horizontal 
position, playing wind instruments, \&c. \&c., a great tendency to reject all fluids through the nares.

A remarkable fact, which should have been noticed before, is that the two sides of the velum approximate each other, or come into contact during efforts at deglutition; it was this fact which first led M. Roux to the idea of uniting them by suture.

Staphyloraphy is an extremely delicate operation; it requires so much docility and patience on the part of the individual operated on that it should not be attempted before the age of fifteen or sixtecn; but this period may be slightly advanced or deferred, according to the determination and courage of the patient. The operation may, indeed, be performed at any period of life; and the earlier it is done the less chance we have of being foiled by a vicious habit of pronunciation; but experience shows that the chances of success are not sufficient to warrant us in undertaking it before the period just mentioned.

In cases of cleft palate complicated with fissure of the hard palate and upper lip, the hare lip should be united at as carly an age as possible.

The instruments employed during the operation are known to all surgeons. They are few and simple; flat ligatures, formed of two or three threads, and fixed in the needles, needle-carriers (porte-aiguilles), hooked forceps, common forceps, a probe-pointed bistoury, and a pair of long-bladed curved scissors, \&c. The most convenient needles are from 0.44 to 0.88 of a line in breadth, slightly enlarged near the eye; of a parabolic form, the curve being 0.88 of a line and the branch which supports the eye being about six or seven lines longer than the other branch.

The patient is placed on a low seat; the ligatures are inserted from below upwards at equal distances; generally three in number, commencing from behind and at from 3.5 to 3.9 lines from the edges of the' fissure. When the point of the needle has passed clearly through, the needle-carrier is opened, and the ligature disengaged by the forceps. The three ligatures being thus placed, the surgeon proceeds to refresh the edges of the fissure. The inferior part of the velum is seized by the hooked forceps, and the excision which has been commenced with the scissors is finished with the bistoury; the flap or portion of tissue thus removed is about 1.7 to 2.2 lines broad. The ligatures are now tied in. the same order that they were placed; the first knot being held by the ring-headed forceps, until the second one is fastened.

Such is the usual course of proceeding, but in cases of division of the hard palate the following modification is made :-When the edges of the fissure have been refreshed, the soft parts are divided transversely by an incision which runs along the posterior edge of the palatine bones. This aids very considerably the extensibility of the two sections of the velum palati, and enables the surgeon to unite them without using the slightest force.

Every circumstance which may impedelthe union of the parts must be carefully avoided. The patient must observe complete silence, and avoid every excitement; he must not swallow his saliva, and even food must be abstained from, the ill-effects of abstinence being counterbalanced, if possible, by nutritious enemata. It must be confessed, however, that the latter are very incfficacious substitutes for food, and few patients can support absolute privation from it. In two cases the want of food gave rise to severe agitation and delirium; on the whole it may be prudent to let the patient have some liquid sustenance in small quantity.

The immediate results of the operation are a sense of tension in the back of the mouth, and sometimes smart pain extending into the ear. The ligatures should be removed on the fourth or fifth day; the inferior ligature the last in cases of simple fissure, but first in the complicated form. Sometimes we can remove the ligatures on the fourth day; sometimes the inferior or superior one is left in for a day longer, according to the distinction just made. The thread is simply taken hold of by a forceps, and cut through. The patient should have some broth before the ligatures are divided. During the next few days he must preserve silence, and take nothing but broth.

The following analysis of nine cases operated on during the course of the year 1841 will serve to furnish some idea of the results of this operation :-

CASE I.-A patient, twenty years of age, had a fissure involving the hard and soft palates and the upper lip. The hare-lip had been operated on some years previously by M. Sanson. A considerable interval separated the two segments of the roof of the mouth in nearly its whole extent; the velum pendulum was thick and strong. The operation was simple: M. Roux divided the velum transversely near the edge of the palatine bones. The patient was examined five months after the oporation, and found to be in a most satisfactory state. The fissure was very considerably reduced, appearing merely as an oval aperturc; he could now speak distinctly, whereas before he could not pronounce words intelligibly.

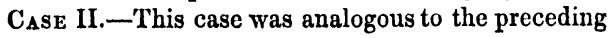
one. M. Roux had operated on the patient for harelip when he was five years old, and the effect of that operation was to diminish the interval between the lateral portions of the hard palate. Ten years afterwards, in April, 1841, staphyloraphy was performed with complete success.

CASE III.-This was the case of a girl, twenty-one years of age. The lip was intact, and the fissure occupied the soft palate only. The operation lasted about half an hour; its results were satisfactory, a small aperture only remaining, which will probably close up with time.

CASE IV.- - Vassel, thirty-one years of age, was admitted into the Hotel-Dieu on the 4th of July, 1841. He was born with simple cleft palate; there is, however, a slight notch in the posterior nasal spine; the lateral segments of the velum are of equal thickness, but the left is narrower than the right.

The operation performed on the 5th of July, presented nothing remarkable, if we except some difficulty in fixing the left portion of the superior ligature. When the threads were drawn together, a small aperture remained, which it was impossible to obliterate by tightening the upper knot. On the following day, in spitc of injunction, the patient talked constantly and drank frequently. On the 9 th the ligatures were removed; union had taken place at a small point only, near the middle of the fissure. This was $\mathbf{M}$. Roux's hundreth operation. 
CASE V. - _ Maret, was admitted into the HôtelDieu on the 8th of July, and the operation performed on the 20th. This was a case of fissure extending through the whole of the palate and upper lip. M. Beclard had operated for the hare-lip when the patient was seven years old, but the operation was only successful in part. The edges of the wound had united by the second intention, but the anterior portions of the hard palate had been so completely approximated, that there was not the slightest interval between the incisor teeth. The left side of the velum was narrower than the right; both were thick and well organised. On the 24 th, the two inferior ligatures were removed; reunion had not taken place at the lowest points. On the 25 th, the superior thread was removed, and here union was perfect. He left the hospital on the 28th.

CASE VI. - A young man, twenty-two years of age, had simple fissure of the velum, with bifurcation of the posterior nasal spinous process. The operation was performed on the 20th of July, 1841. The two superior ligatures were removed on the fourth day, and the inferior one on the fifth. Complete union had taken place, but the cicatrix gave way opposite the lower thread, in consequence of violent coughing. There likewise remained a very small oval aperture from the bifurcation alluded to. On the 13th of August it became necessary to apply a fresh ligature over the inferior portion of the velum.

Case VII._- Mas, twenty years of age, a musician, was operated on at the Hotel-Dieu, on the 24th of August. The case was one of simple fissure of the soft palate. Four days afterwards the ligatures were removed and complete union had taken place. The cicatrix was firm on the 29th, when the patient was allowed to speak and eat. On the 3lst nothing remained, except an oval aperture of 0.8 to 1.3 of a line in diameter, from bifurcation of the posterior nasal process.

CASE VIII.-The operation in this case was performed in London, in the month of September, 1841, on a man, thirty-five years of age, not for congenital malformation, but for loss of substance from syphilitic ulceration. The operation failed.

CASE IX.-This operation also failed; it was performed on a tailor, named Hurel, eighteen years of age. The velum palati was thick and short, divided in its whole length; the posterior moiety of the hard palate was likewise fissured. On the 1st of November, 1841, two sutures only were inserted, as the palate was narrow; the edges of the wound were easily refreshed and the transverse incision made without difficulty. There was some trouble in tying the inferior knot, because the uvula was constantly driven against it, by the base of the tongue. The two threads were removed on the 5 th, when it was found that the middle portion of the wound only had united. The voice, however, was less disagreeable. On the 7 th, the whole of the cicatrix gave way while the patient was coughing.

From the preceding observations, it appears that of the nine cases just related, five were examples of complete and complicated fissure of the palate, cases in which the prognosis is always unfarorable. In three of those five cases the result was as favorable as could be expected; in one of the remaining two the operation completely failed; in the other a partial benefit resulted. Four cases were examples of simple fissure, although in two there was bifurcation of the posterior spinous process, which is always followed by the persistence of a small aperture. This latter can only be removed by having recourse to palatoplasty, and seldom if ever closes of its own accord. Finally, of two of the most simple cases, one failed, while the other was only partially successful. Of the nine patients operated on, one was a female; their ages varied from 15 to 35 ; the majority of cases ( 5 in 9 ) occurred between 20 and 22 years.

Although the operation is, in itself, attended with no danger, yet in 3 of the 105 cases it was followed by death. In one, severe inflammation of the pharynx set in, and extended to the air-passages. The second was a young Englishman, who died with irregular nervous symptoms. The last case occurred in the daughter of an eminent English nobleman, who was carried off soon after the operation by phthisis. In $1828, M$. Roux had performed 40 operations for cleft palate; 19 were cases of simple fissure, and of these 13 were successful, 6 unsuccessful; 21 were cases of complicated fissure, of which 12 failed, and 9 succeeded. In 1834 he had performed 68 operations; in $1842,105$.

The general result was, success in two-thirds of the cases of simple fissure; and in one-third only for cases of complicated fissure. This is not so favorable a result as that obtained from the earlier operations, but as it is drawn from a large number of cases, it probably gives a fair estimate of the chances of success or failure of the operation.-Gaz. Med. de Paris, No. 30.

\section{PROVINCIAL MEDICAL JOURNAL}

\section{SATURDAY, DECEMBER 24, 1842.}

Not the least valuable among the results of the registration is the prospect afforded of attaining through the returns some knowledge of the laws which regulate the development and progress of epidemic disease. We have before had occasion to direct attention to the prevalence of different diseases of this character at different times. The second report of the registrar-general shows, that although various affections belonging to the epidemic class are coexistent at the same time in different localities, there is yet a general rise, prevalence, and decline of each occurring at different times, and apparently following in their progress certain definite laws throughout the whole extent of country embraced in the returns. An additional confirmation is thus afforded to the accuracy of the ancient writers in matters of observation; and the dissertations of Sydenham, on the cpidemic constitution of the atmosphere at particular times and seasons, derive fresh interest and importance.

The influence of climate, season, age, sex, temperament, occupation, and local peculiarities of situation, 